\title{
Robot-aided Tunnel Inspection and Maintenance System
}

\author{
J. C. González, S. Martínez, A. Jardón and C. Balaguer \\ Roboticslab Engineering Systems and Automation Department, Universidad Carlos III de Madrid, 28911 \\ Leganés, SPAIN, \{jcgvicto, scasa, ajardon, balaguer\}@ing.uc3m.es
}

\begin{abstract}
This paper describes an innovative alternative to manual procedures for the application of carbon fiber and resin injection in concrete surfaces in tunnels. It is based on a specially designed light-weight integrated tool for automatic application of Fiber Reinforced Polymer (FRP) and epoxy resin injection. Vision and laser telemeter sensing are integrated into the tool to assure precise inspection and maintenance operations. An interconnection flange allows simple and robust attachment to a robotic manipulator's tip. The robot-tool set is mounted on an articulated lift platform. Therefore, an operator can direct the platform and the robottool's operations in a control station placed at ground-level, in a wheeled vehicle on which the articulated lift platform is mounted. An intuitive Human-Machine Interface (HMI) has been developed to allow the operator to identify fissures for the injection of epoxy resin, and to choose where to place the FRP strips. Actual procedures are completely automatic.
\end{abstract}

Keywords: Robotic automation, Robotic tool design, Tunnel maintenance, Concrete inspection, HMI

\section{Introduction}

Tunnels nowadays are designed and built to last hundreds of years. However, change in use, new load criterions, and impact and damage caused by natural and human factors can reduce service life to less than a tenth of its original estimate.

Inspection and maintenance operations in tunnels depend heavily upon time and space constraints, the tunnel's intrinsic conditions (reduced space, possible existence of service pipes), traffic flow, and, in railway tunnels, the presence of aerial electric cable. Working conditions in these subterranean infra- structures can be slow and tedious due to dust, humidity, complete absence of natural light, and uncomfortable working conditions. Efficiency and stigma of any operator gradually reduce throughout the day, incrementing the risk index. Any attempt to automate operations performed in subterranean infrastructures will drastically improve short and long-term security, and increase productivity [1].

A great range of factors can cause need for maintenance or reparation operations. Two of the most important of these factors will be treated:

- Fissure formation due to deformation caused by excessive load. Although current legislations tolerate the existence of small fissures, their real dimensions should never exceed a small, reduced range. Reinforced concrete's interior metal infrastructure should never be exposed to ambient atmosphere.

- Loss of quality of the infrastructure's surface due to lack of coating, needed to guarantee correct metal-conglomerate adhesion. Most pathological phenomena (carbonation, chlorine...) pass undetected until external signs appear on the iron structure: rust, section and adhesion reduction...

\section{Maintenance Operations}

Presently, all inspection and maintenance operations in tunnels are performed manually (Figure 1). Frequently, traffic flow must be cut, and scaffolds mounted, implying the subsequent loss of global productivity. Maintenance operations involve the following set of tasks: superficial preparation, fissure injection, and FRP composite adhesion.

\subsection{Superficial Preparation}

This includes all of the processes needed to eliminate concrete in bad state. Loss of mechanical capacity 
or of stability within the rest of the structure can be a possible reason for this need. The surface must be prepared before concrete reparation and/or restoration. Common methods include compressed air blowing, sand abrasion, and hydro-demolition (preparation with high-pressured water).

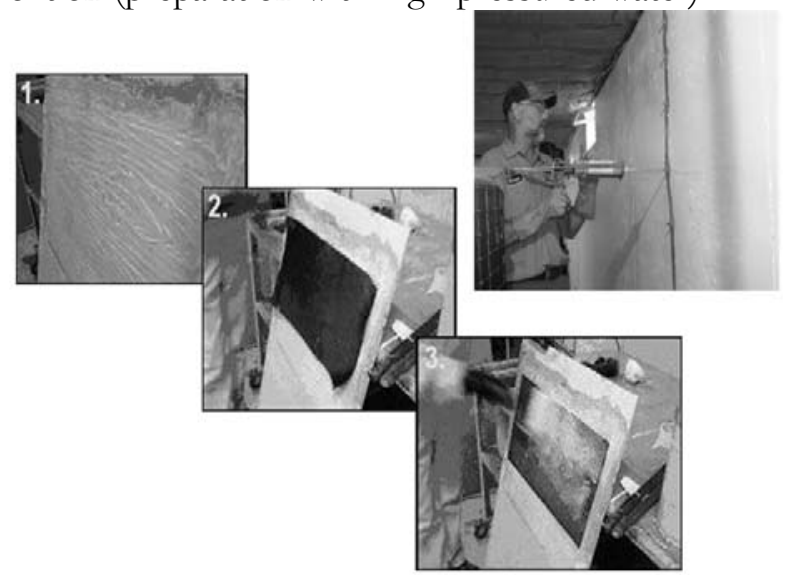

Figure 1. Conventional Manual Procedures

\subsection{Fissure Injection}

Development of advanced materials such as low-viscosity epoxy resins allows pressure injection of these materials through fissures as small as $0.2 \mathrm{~mm}$ thick. They are usually thixotropic, solvent-free, and bicomponent. Components are included in different compartments of a same cartridge, and combined through a static mixer at application time. The main objective of these injections is to re-establish continuity in the concrete section. Injections can be used to fill in internal or hard-to-access zones (such as internal nests). This technique has been used and tested on tunnels, bridges, and several nuclear centrals over the past few years.

\subsection{FRP Composite Adhesion}

Since its first applications in Europe and Japan in the 1980s, superficial reparation and restoration with Fibre Reinforced Polymer (FRP) has progressively increased. Quick and easy installation and high versatility make FRP reparations attractive to owners, engineers and promoters. Carbon or aramid fibres allow high mechanic, thermal, electric and chemical resistance, high modulus of elasticity, and low density [2]. Forms of interest include flexible strips that can be adhered to concrete surfaces using application-specific epoxy resins.

The following considerations should be taken into account when applying FRP to external surfaces:

- Dimension and orientation of each strip.

- Number of strips and sequence of installation.

- Time limits between successive strips.

- Surface temperature and moisture limitations.

- General notes listing design loads and allowable strains in the FRP laminates.

The main strength component of a non-slender, normal-weight concrete member confined with an FRP jacket may be calculated using the FRP confined concrete strength equation (1) or (2).

$$
\begin{aligned}
& \phi P_{n}=0.85 \phi\left\lfloor 0.85 \psi_{f} f_{c c}^{\prime}\left(A_{g}-A_{s t}\right)+f_{y} A_{s t}\right\rfloor \\
& \phi P_{n}=0.80 \phi\left\lfloor 0.85 \psi_{f} f_{c c}^{\prime}\left(A_{g}-A_{s t}\right)+f_{y} A_{s t}\right\rfloor
\end{aligned}
$$

Confining a concrete surface is accomplished by orienting the fibres transverse to the weakest axis of the member, resulting in an increase in the apparent strength of concrete and in the maximum usable compressive strain [3]. In this orientation, fibres work similarly to conventional spiral or tie reinforcing steel. However, any contribution of the longitudinally aligned fibres to the axial compression strength of a concrete member should be neglected, as fibres are usually displaced in a unidirectional fashion, following the direction of the strip or roll. FRP jackets provide passive confinement, remaining unstressed until dilation and cracking of the member occurs [4]. For this reason, intimate contact between FRP and concrete surface is critical.

FRP adhesion is also used in automotive, marine, and aerospace industries. 


\section{Integrated Process Automation}

The Robot Aided Tunnel Inspection and Maintenance (RATIM) System is the result of the first attempt ever to automate superficial preparation, fissure injection, and FRP composite adhesion procedures. An integrated system was mounted with a specially designed tool, a robot arm, and a wheeled vehicle with a platform lift (as seen in Figure 2). This configuration has also been applied with Kuka robots in Korea [5], but never before for concrete reparation process automation.

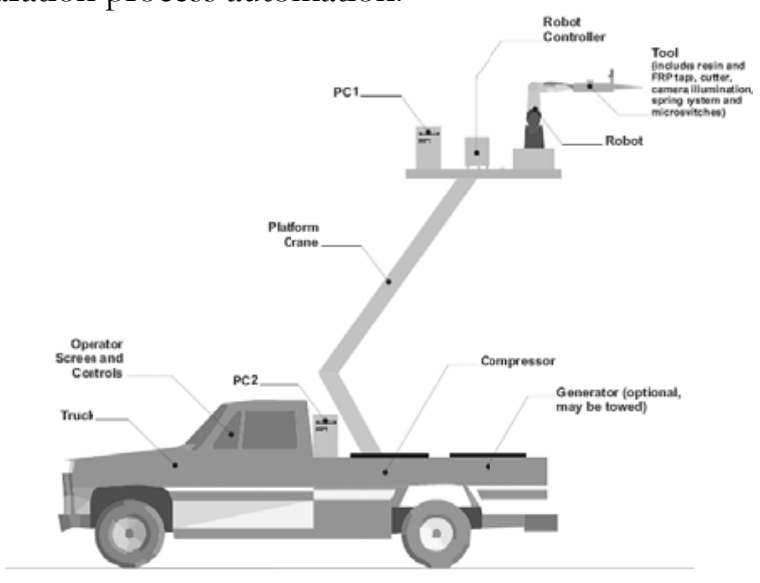

Figure 2. Basic Hardware Architecture

Inspection is performed through a user-friendly guided HMI, where camera image stream is displayed and operation procedures are solicited. Visual servoing based on the depth measure captured by the tool's laser telemeter, and operation-oriented actuators are coordinated through task-specific control software [6].

For precise operation, the robot arm chosen for the application was the Mitsubishi PA-10, a 7 DOF manipulator with a $10 \mathrm{~kg}$ load capacity and a 1 meter maximum extension range. The global increment of this range is achieved by mounting the Robot-Tool a 5 meter extensible articulated lift platform. The HMI is installed in the wheeled vehicle's cabinet to which the articulated lift platform is attached. Power for the system can be supplied from an on-board generator, the wheeled vehicle's motor, or the tunnel's basic provided services.

When the surface to treat is inside the reach volume, user extends the vehicle's stabilizers, directs the platform towards the concrete surface, and then follows the HMI's on-screen instructions.

\section{Specially Designed Tool}

A light-weight sensed integrated tool was designed and manufactured for the automation of the described processes. Material provider's workshops and real construction sites were visited to analyze manual procedures 'in situ'. Conclusions were that radical and innovative approaches would have to be taken to lead this system to complete automation.

- Minimum changes should be needed to be effectuated on the tool to change between superficial preparation and resin injection process, and superficial preparation and FRP adhesion process.

- A three-in-one, one-pass FRP adhesion process (resin, FRP, resin) is desirable.

- Maintaining the weight of the tool low throughout the design process would allow it to be attached and used within a wide range of commercial and non-commercial robots.

- The robot-tool interconnection flange must allow simple mounting and un-mounting.

- Vision, laser distance, and security systems should be intrinsic to the tool.

Objectives were achieved, allowing the tool to benefit from the advantages of repeatability, reliability, and precision provided by any robot. Conceptual design can be seen in Figure 3.

The final designed tool is composed by two complementary systems: a material application system (mechanical subsystems and actuators), and a vision and security system (camera, laser distance sensor, and security micro-switches). 


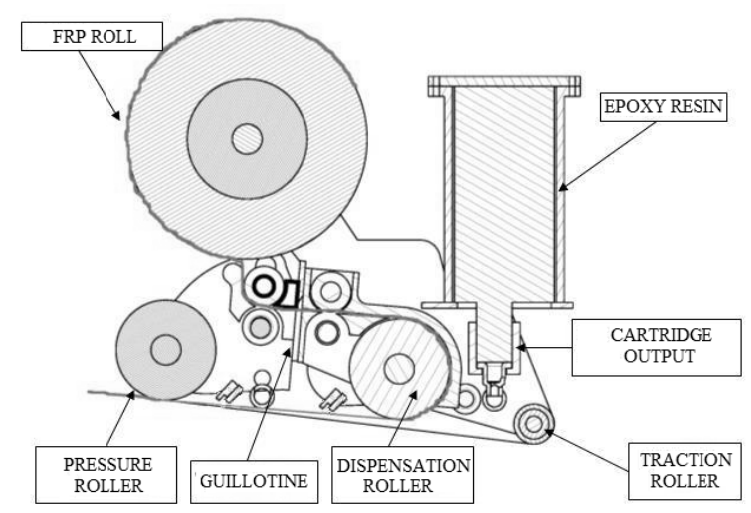

Figure 3. Tool's Basic Conceptual Design

Compressed air blowing was the option chosen for superficial preparation. Sand abrasion or hydrodemolition would have made sand or water available too, thus reducing system flexibility. The availability of compressed air is achieved with an on-board compressor mounted on the wheeled vehicle, though most tunnels also provide this service. Air flow is digitally controlled by system-mounted mini-electrovalves. Availability of compressed air also led us to one of the system's fundamental breakthroughs: Resin cartridges' push is triggered by canalization of compressed air towards its piston (Figure 4), therefore obviating the need of a motor, gear reductions, and additional mechanical components. This frees the tool from extra weight, size, and cost.

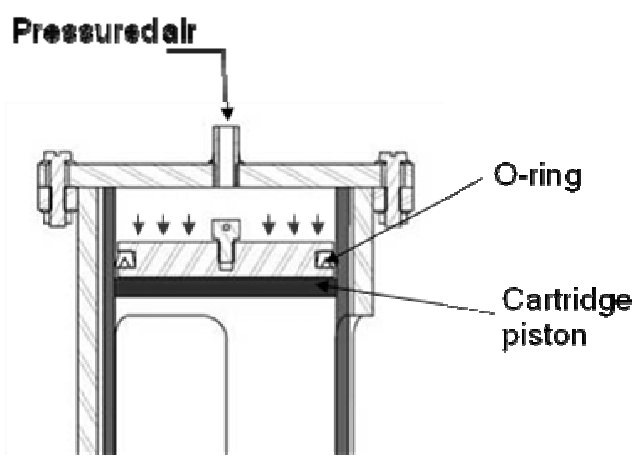

Figure 4. Resin Activation Mechanism

A sophisticated gear-and-roller-conjunction system was designed for the dispensation of FRP strips. Commercial FRP is distributed in the form of a long roll. In order to cut the roll into strips of the desired length, the gear-and-roller system is combined with a cutting mechanism that is activated by relative movements between the tool's internal mobile parts and the tunnel's surface. Linear actuators (pneumatic cylinders) were incorporated to automate the cutting process without having to depend on the robot or manipulator's strength against this surface. This is beneficial for the weaker range of manipulators. A linear dispenser, similar to the one used for air in the superficial preparation process, was designed to spread resin over the tunnel's weakened surface. Pre-impregnated FRP rolls were discarded due to possible undesired FRP-tool interaction due to adhesive properties. In the designed system, rollers, gears, and resin dispensation work simultaneously as the tool advances to obtain a compact resin-FRP-resin sandwich mounted on the tunnel's surface (Figure 5).

An Axis IP surveillance camera and an ultra-light precision Laser Telemeter sensor are the two main components of the vision system. Sensor ray and the camera' central pixel point are oriented parallel to the axis of the resin cartridge. Stereo vision was discarded due to its ineffectiveness on homogeneous patterns, such as tunnel concrete surfaces. The IP Surveillance Camera obtains images with a streaming resolution up to $1280 \times 1024$ pixels and 30 frames per second, sufficient for having real-time visual information in a control cabin. Micro-switches activated by contact with the tool's mobile parts assure mechanical safety. 


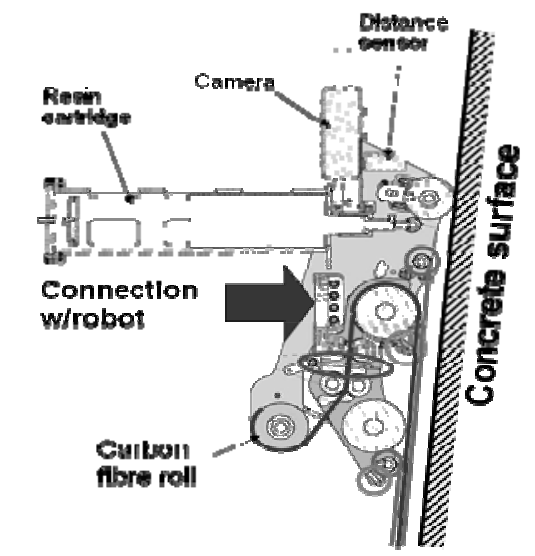

Figure 5. FRP Application Scheme

\section{HMI and Control Software}

Both HMI and control software were coded entirely in $\mathrm{C}++$, a powerful multi-platform object-oriented programming language. The user-friendly HMI guides the system operator intuitively through the inspection and identification tasks (Figure 6), for superficial preparation and epoxy resin injection, or superficial preparation and FRP adhesion.

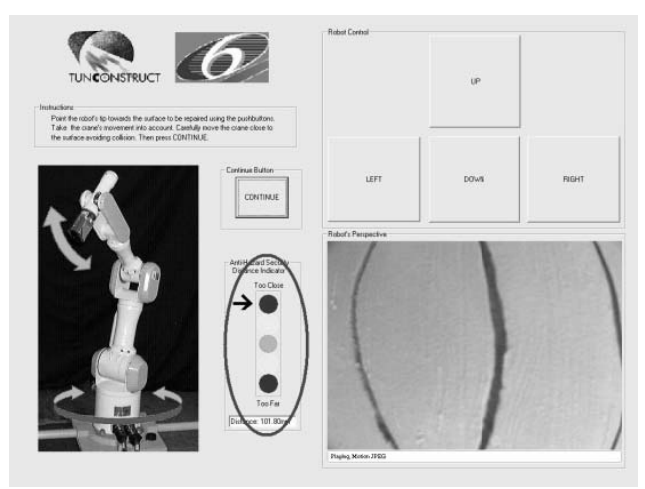

Figure 6. Intuitive HMI w/Distance Indicator

A virtual traffic light warns user if he or she is putting any part of the system's integrity in risk. (Figure 6, circled red). Both procedures converge into the final step: pressing a simple 'perform automatic operation' screen button. Previsualization of the desired process is also included in the software pack (Figure 7).

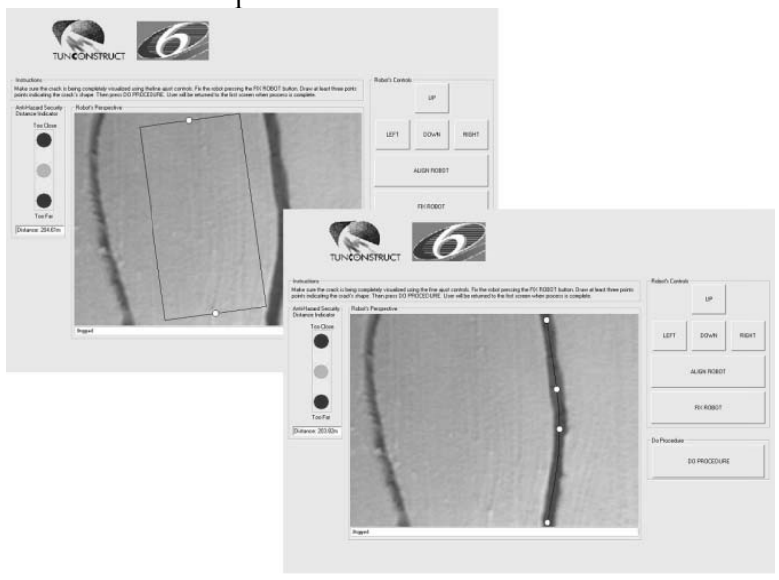

Figure 6. Intuitive Guided HMI Screenshots

Internally, software parameters are set in headers, and additional calibration information is calculated at run-time. Reusable spline and geometrical calculation libraries, developed specifically for this innovative robotic application, are integrated into the software. Barrel-type image distortion is corrected using the 
previously calculated intrinsic parameters of the camera. Communication schemes between HMI and control software are implemented over TCP/IP, the Internet Protocol. This means the operator could, technically, run the HMI software to identify fissures and weakened surfaces from any place in the world.

\section{Laboratory and Field Tests}

The RATIM was first tested indoors, using models manufactured by FRP and epoxy resin providers (Figure 8). Models were disposed in horizontal, vertical, and intermediate positions to assure material consistency (resin dripping would have been undesirable), robot configuration singularity avoidance [7], as well as correct robot-tool interaction (collision avoidance) [8].

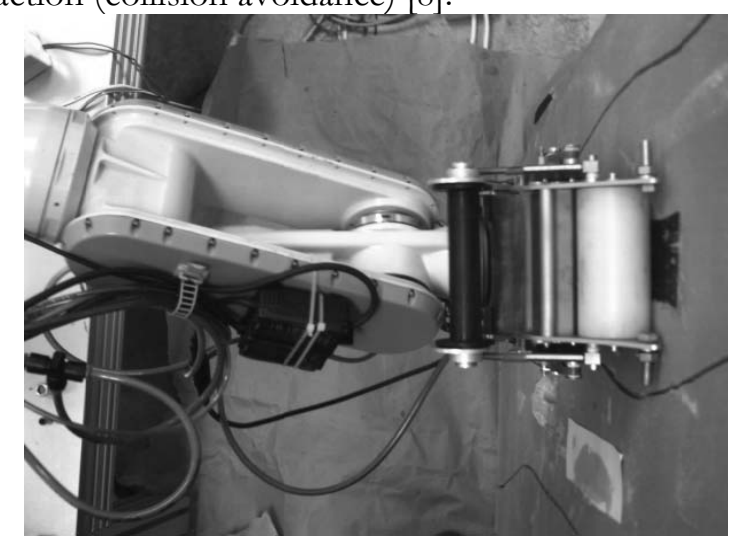

Figure 7. Laboratory Tests at UC3M

After having succeeded in proving effectiveness in laboratory tests, the complete integrated system was tested outdoors. Demonstrations were performed in real, non-controlled environments in tunnels in Bembibre, León (Figure 8).

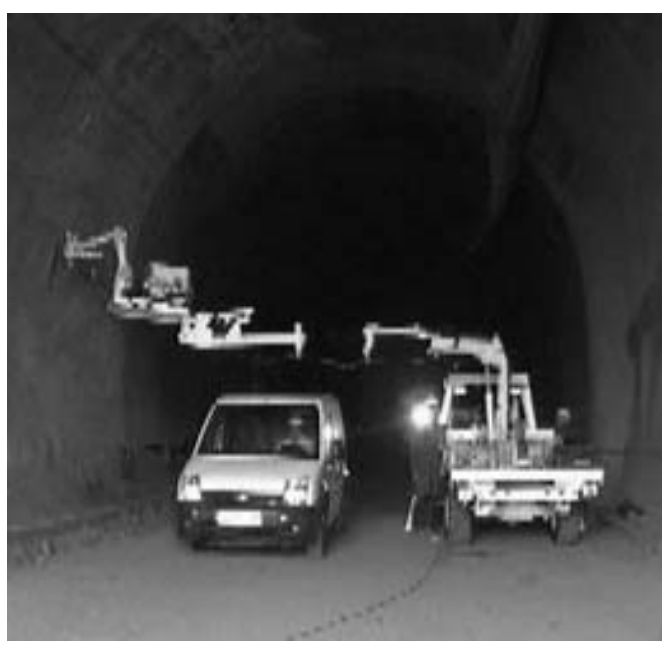

Figure 8. RATIM System Tested Outdoors

There too, tests provided satisfactory results. Test samples were extracted from the tunnel's treated surfaces. Stress and strength parameters, contrasting equation (1) results, were analysed and validated at Sika S.A.U.'s laboratories.

\section{Conclusions}

The Robot-Aided Tunnel Inspection and Maintenance System is definitively the first system of its kind, a precedent for any robotic tunnel inspection and maintenance system of the future. It has been developed for inspection, maintenance, and reparation of small fissures and weakened surfaces. This fulfils the philosophy of true preventive maintenance. Reparation of small fissures beforehand will prevent the evolution of these 
small fissures into great, steep cracks. Just as well, reinforcement of weakened structures will prevent solicitations that can provoke the deterioration of the rest of the tunnel's infrastructure. Main characteristics of the RATIM system include reduction of operation times, a quick system and HMI learning curve, and a global increase of commodity and productivity for operator and society. Operation can be achieved, risk free, without need of stopping traffic flow or mounting colossal scaffolds.

\section{References}

[1] C. Balaguer (2000) Open issues and future possibilities in the EU construction automation IAARC ISARC '00, Taipei, Taiwan, K21-32.

[2] Nanni A.; Bradford N.M. (1995) FRP Jacketed Concrete under Uniaxial Compression, Construction and Building Materials, Vol. 9, No. 2, 115-124.

[3] H. Toutanji (1999) Stress-Strain Characteristics of Concrete Columns Externally Confined with Advanced Fibre Composite Sheets, ACI Materials Journal, Vol. 96, No. 3.

[4] M. R. Spoelstra; G. Monti (1999) FRP- Confined Concrete Model, Journal of Composites for Construction, Vol. 3, No. 3, 143-150.

[5] S. Lee; M. Gil; K. Lee; J. Lee; C. Han (2007) Design of a Ceiling Glass Installation Robot, IAARC ISARC '07, Vol 2.4, 247-252.

[6] A.Giménez; J.M.Pastor; V.M.Padrón; M.Abderrahim (2002) Unmanned Vehicles for Aerial, Ground and Naval Military Operations. Elsevier, NATO RTO series, No. 52, 161-166.

[7] C. Balaguer (2006) Robótica y Automatización, Fundación COTEC, ISBN: 84-95336-61-8.

[8] L. Moreno, S. Garrido, C. Balaguer (2003) Ingeniería de Control. Modelado y Control de Sistemas Dinámicos. Ariel, ISBN: 84-344-8055-7. 